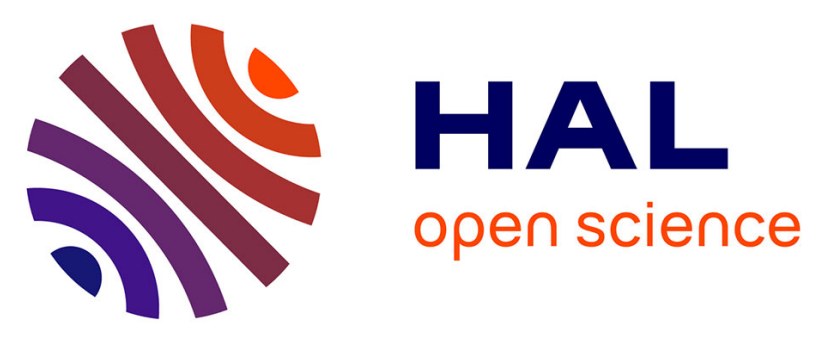

\title{
Revealing Crystallization Dynamics and the Compositional Control Mechanism of 2D Perovskite Film Growth by In Situ Synchrotron-Based GIXRD
}

Liang Kuai, Junnan Li, Yajuan Li, Yusheng Wang, Pandeng Li, Yuanshuai Qin, Tao Song, Yingguo Yang, Zhuoying Chen, Xingyu Gao, et al.

\section{To cite this version:}

Liang Kuai, Junnan Li, Yajuan Li, Yusheng Wang, Pandeng Li, et al.. Revealing Crystallization Dynamics and the Compositional Control Mechanism of 2D Perovskite Film Growth by In Situ Synchrotron-Based GIXRD. ACS Energy Letters, 2020, 5 (1), pp.8-16. 10.1021/acsenergylett.9b02366 . hal-02989846

\section{HAL Id: hal-02989846 https://hal.science/hal-02989846}

Submitted on 21 Nov 2020

HAL is a multi-disciplinary open access archive for the deposit and dissemination of scientific research documents, whether they are published or not. The documents may come from teaching and research institutions in France or abroad, or from public or private research centers.
L'archive ouverte pluridisciplinaire HAL, est destinée au dépôt et à la diffusion de documents scientifiques de niveau recherche, publiés ou non, émanant des établissements d'enseignement et de recherche français ou étrangers, des laboratoires publics ou privés. 


\section{Revealing Crystallization Dynamics and Compositional Control Mechanism of}

2D Perovskite Film Growth by In-situ Synchrotron-based GIXRD

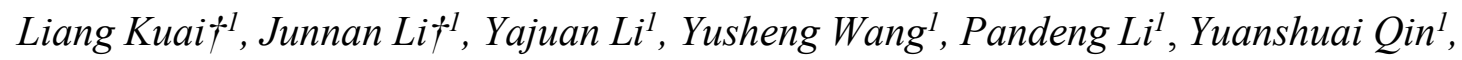
Tao Song ${ }^{1}$, Yingguo Yang ${ }^{2 *}$, Zhuoying Chen ${ }^{3}$, Xingyu $\mathrm{Gao}^{2}$, Baoquan Sun ${ }^{1 *}$

${ }^{1}$ Institute of Functional Nano \& Soft Materials (FUNSOM), Jiangsu Key Laboratory for Carbon-Based Functional Materials \& Devices, Soochow University, 199 Ren'ai Road, Suzhou, 215123, Jiangsu, PR China

${ }^{2}$ Shanghai Synchrotron Radiation Facility (SSRF), Zhangjiang Lab., Shanghai Advanced Research Institute, Chinese Academy of Sciences, 239 Zhangheng Road, Shanghai 201204, China

${ }^{3}$ LPEM, ESPCI Paris, PSL Research University, Sorbonne Université, CNRS, 10 Rue Vauquelin, 75005 Paris, France

$\dagger$ Both authors have equal contribution to this work. *Corresponding author: yangyingguo@sinap.ac.cn,bqsun@suda.edu.cn 


\section{Abstract}

Quasi-two-dimensional (2D) perovskites promise the intrinsically stable solar cell performance. However, the crystal orientation and phase distribution in 2D solution processed perovskite are difficult to be manipulated, which restricts the device efficiency as well as its reproducibility. Here, we simply incorporate potassium ion $\left(\mathrm{K}^{+}\right)$into quasi-2D precursor solution, which can dramatically change the nucleation steps during perovskite films spin-coating process probed by in-situ synchrotron-based grazing incident X-ray diffraction (GIXRD). It is notable that a desired vertical oriented 2D phase without intermediate compound can be easily formed after spin-coating, which simultaneously reduces the distribution of low dimensional 2D perovskite phases in association with suppressed trap states. Therefore, the power conversion efficiency of doped $10 \%$ $\mathrm{K}^{+} 2 \mathrm{D}$ perovskite solar cells can yield up to $11.3 \%$ as well as long-term stable performance with high reproducibility. This work paves a key path to control the quasi-2D nucleation and crystallization processing via chemical additives. 
In recent years, organic-inorganic hybrid lead halide perovskites have drawn wide attentions due to its outstanding photoelectric properties..$^{1-5}$ The perovskite material has widely applied to solar cells,,${ }^{1,6}$ light-emitting diodes (LED), ${ }^{7-8}$ and photodetectors. ${ }^{9-10}$ The power conversion efficiency (PCE) of three-dimensional (3D) perovskite solar cell has raised up to $25.2 \%,{ }^{11}$ however, the stability issues still retard the future development including the temperature degradation, humidity degradation and ultraviolet lightinduced degradation. ${ }^{12-15}$ In order to improve 3D perovskite material stability, interfacial modification has been considered as an efficient method to prevent the degradation of perovskite solar cells. ${ }^{16-19}$ Except for the optimization of interface engineering, the moisture sensitivity of perovskite material can also be crucial for device resultant stability. ${ }^{20-22}$ Dimensionality engineering has drawn consideration attention to improve devices stability by structural manipulation. ${ }^{23-25}$ Two-dimensional (2D) halide perovskite has been recently considered as more stable materials than 3D ones. Meanwhile, 2D perovskite plays crucial roles on photovoltaic performance, which can be used as pure light absorber, ${ }^{26-29}$ capping layer, ${ }^{30-31}$ passivation layer or mixed 2D/3D light absorber. ${ }^{32-36}$ The application of 2D perovskite can obviously improve the stability and performance of perovskite solar cells.

Among the multiple application, quasi-2D Ruddlesden-Popper (RP) perovskite can be formed by incorporating organic long chain molecules, ${ }^{37-38}$ which can retard the 3D perovskite crystal growth. Meanwhile, hydrophobic organic molecules could protect perovskite from moisture intrusion. The $2 \mathrm{D}$ RP structure can be regarded as the dimensional reduction of $3 \mathrm{D}$ perovskite, which has the chemical formula of $\mathrm{A}_{2} \mathrm{~B}_{\mathrm{n}}$ 
${ }_{1} \mathrm{M}_{\mathrm{n}} \mathrm{X}_{3 \mathrm{n}+1}$, where $\mathrm{A}$ represents organic ligand, such as phenethylammonium ( $\mathrm{PEA}^{+}$), butylammonium $\left(\mathrm{BA}^{+}\right)$; B represents monovalent cation, such as formamidinium $\left(\mathrm{FA}^{+}\right)$, methylammonium $\left(\mathrm{MA}^{+}\right)$; $\mathrm{M}$ represents divalent metal ion, such as lead $\left(\mathrm{Pb}^{2+}\right)$, tin $\left(\mathrm{Sn}^{2+}\right)$; and $\mathrm{X}$ stands for halide ion, such as $\mathrm{I}^{-}, \mathrm{Br}^{-}$and $\mathrm{Cl}^{-} . n$ represents the dimensional thickness of the inorganic layers, which affects the optical and electronic properties of quasi-2D RP perovskite. In quasi-2D RP perovskite, the exciton binding energy is enhanced due to the dielectric confinement effect between organic layer and inorganic layer, and the charge transport also be suppressed on account of the additional introduction of bulky organic ligands.

Recently, considerable works have found that the quasi-2D perovskite at room temperature can always form different phase distribution due to the rapid reorganization process even though the precursor solution is prepared with particular nanoplatelet thickness ( $n$ value).$^{39-40}$ Transient absorption spectroscopy indicates that the electrons and holes can incur funnel effect, where energy can transfer from low $n$-number to high $n$ ones. ${ }^{41-42}$ Different nanoplatelet thickness distributions would affect the funnel effect. Beside the phase distribution issue, it has been found that when the crystal growth orientation is parallel to substrate, it would hinder charge transport in the out-of-plane direction due to the insulating organic layers, which affects device performance. ${ }^{43}$ Therefore, both the crystal orientation and phase distribution are crucial to improve the performance of quasi-2D RP perovskite solar cells.

In order to solve the above-mentioned issues of parallel orientation and mixed phase distribution in quasi-2D RP perovskite, Tsai et al. found that thin film morphology 
appears near-single-crystal quality by hot-cast technique, more delightedly, the (001) and (010) planes of inorganic perovskite component display remarkable out-of-plane direction relative to substrate, which facilitates efficient charge transport. The PCE of $\mathrm{BA}_{2} \mathrm{MA}_{3} \mathrm{~Pb}_{4} \mathrm{I}_{13}$ composition drastically increased from initial $4.02 \%$ to $12.5 \%{ }^{29}$ Although the efficiency was enhanced obviously, the mechanism and orientation control has not been fully understood until recently Chen et al. revealed that the nucleation and vertical crystal growth incurs in the liquid-air interface. ${ }^{43}$ Unfortunately, hot-cast technique is difficult to be manipulated because the substrate requires a quick transfer from a hot plate to cold spin-coater chuck in order to maintain the substrate temperature, therefore, the device exhibits poor reproducibility. Liu et al. utilized mixed solvent of dimethyl formamide (DMF) and dimethyl sulfoxide (DMSO), which can also form desired vertical orientation. ${ }^{39}$ However, the method tends to form multiple phase distribution due to slow down the crystallization, which results in inferior PCE of $8.54 \%$. Alternatively, chemical additives are likely to control the growth orientation and improve crystallization quality, such as ammonium thiocyanate $\left(\mathrm{NH}_{4} \mathrm{SCN}\right)$, ammonium chloride $\left(\mathrm{NH}_{4} \mathrm{Cl}\right)$, methylammonium chloride $(\mathrm{MACl}){ }^{28,}{ }^{44-45}$ However, the incorporation of chloride ion requires the regulation of anti-solvent, which increase the complexity of processing. Recently, alkali metal doping in 3D perovskite has drawn tremendous attention, it can obviously improve both the performance and stability of solar cells. Our previous work also revealed that incorporating potassium ion $\left(\mathrm{K}^{+}\right)$into 3D precursor solution can form new potassium-rich phase in grain boundary, which 
reduced the trap densities. ${ }^{46}$ However, based on our best knowledge, there is no research on small alkali metal doping in quasi-2D perovskite.

Here, $\mathrm{K}^{+}$was used to align the $2 \mathrm{D}$ perovskite layers perpendicular to the substrate as well as tune the nanoplatelet phase distribution. It was found that the doped $\mathrm{K}^{+}$quasi2D perovskite film achieved desired vertical orientation via in-situ GIXRD and $\theta-2 \theta$ X-ray diffraction $(\mathrm{XRD})$ characterizations during spin-casting at room temperature without any other further post-treatments. Notably, compared with DMSO solvent method and hot-cast technique, the addition of $\mathrm{K}^{+}$can directly convert to large crystallite size $2 \mathrm{D}$ phase without intermediate compound and suppress the formation of low $n$-number phase $(n<4)$, which is favorable for preparing highly efficiency and stability quasi-2D perovskite solar cells. Therefore, the optimized $10 \% \mathrm{~K}^{+}$doped solar cell can achieve the PCE of $11.3 \%$ with excellent repeatability. In addition, the unencapsulated devices can sustain $90 \%$ of initial performance after 1000 hours in $40 \% \pm 10 \%$ relative humidity at room temperature. Our results provide new insight into morphology, crystallinity, and orientation of quasi-2D perovskite targeting highly efficient and stable device.

Following previously reported method, ${ }^{39}$ quasi-2D perovskite with $\mathrm{BA}_{2} \mathrm{MA}_{3} \mathrm{~Pb}_{4} \mathrm{I}_{13}$ composition was fabricated via dissolving $1 \mathrm{M} \mathrm{PbI}_{2}, 0.75 \mathrm{M}$ methylammonium iodide (MAI) and $0.5 \mathrm{M}$ butylammonium iodide (BAI) in DMF as the reference device. The films without and with $\mathrm{K}^{+}$are defined as BAMA $\left(0 \% \mathrm{~K}^{+}\right)$and KBAMA, respectively. To fabricate KBAMA quasi-2D perovskite films, KI is incorporated into BAMA perovskite precursor solution with different molar ratios $\left(x \%=M_{\mathrm{KI}} / M_{\mathrm{BAMA}}\right)$ from $5 \%$ to 
$20 \%$. Meanwhile, in order to compare the properties of quasi-2D perovskite films with different processing conditions, we also fabricate the films with DMSO solvent and adopting hot-cast technique. ${ }^{29,39,47}$ The detailed experimental information can be found in Supporting Information ST1. To investigate the morphology of films under different treatment conditions, we employ the scanning electron microscopy (SEM) characterization, as shown in Figure 1(a)-(d). When DMF is used as solvent and the film is spin-coated at room temperature (DMF RT), there are some pinholes in the film, and the film is discontinuous due to the rapid crystallization. ${ }^{48}$ When DMSO instead of DMF is used as solvent (DMSO RT), the crystal shape turns to be typical flake and the film becomes more continuous due to the reduced crystallization rate. Interestingly, when $10 \% \mathrm{~K}^{+}$is incorporated, the film morphology becomes smooth without any pinholes in association with few crystalline cracks, which is as similar as that by hotcast technique. Continuous film would benefit charge transport and reduce trap states, which is favorable to enhanced performance of perovskite solar cells. When different concentration $\mathrm{K}^{+}$is incorporated, the corresponding SEM images are shown in Figure S1. There are still some pinholes when only $5 \% \mathrm{~K}^{+}$is incorporated. Further increasing the concentration of $\mathrm{K}^{+}$beyond $10 \%$, the film morphology quality becomes worse, especially when doped $20 \% \mathrm{~K}^{+}$, there are many large pinholes on the surface. The excess $\mathrm{K}^{+}$likely acts as impurity, which may retard the perovskite crystallization. In order to investigate the distribution of $\mathrm{K}^{+}$in quasi-2D perovskite film, we conduct energy-dispersive X-ray spectroscopy (EDX) mapping of 10\% KBAMA film, as shown in Figure S2. $\mathrm{K}^{+}$is homogenously distributed in the quasi-2D perovskite film, which 
is as similar as $\mathrm{Pb}^{2+}$ and $\mathrm{I}^{-}$. The phenomenon is different from our previous work in doped $\mathrm{K}^{+} 3 \mathrm{D}$ perovskite, where $\mathrm{K}^{+}$is only located at grain boundary. ${ }^{46}$

In order to investigate the crystal growth orientation of quasi-2D perovskite films with different processing conditions, the GIXRD characterization is conducted, as shown in Figure 1(e)-(h). The crystal growth orientation with varied processing conditions are different, which is obviously showed in Figure 1(i)-(j). The DMF RT film shows powder-like rings in the diffraction pattern indicating the grains are randomly oriented, which is as similar as 3D perovskite crystal. The DMSO RT film shows sharp and discrete Bragg spots, which indicates the quasi-2D crystals predominately orient vertically. Simultaneously, sharp (0k0) peaks appear at small angle, which belong to a mixture of low $n$-number phases. ${ }^{49-50}$ The film fabricated with $10 \% \mathrm{~K}^{+}$additive exhibits the sharp, sporadic Bragg spots, suggesting highly vertical oriented growth, being consistent with hot-cast technique. In addition, the peaks for low $n$-number phases disappear in the GIXRD pattern, which would indicate that $\mathrm{K}^{+}$incorporation hinders the formation of lower $n$-number nanoplatelet phases in association with promoting the desired oriented quasi-2D phase. This result is astonishing, as vertical orientation and organized phase in quasi-2D perovskite film are widely convinced to be favorable for solar cell performance.

The $\theta-2 \theta$ XRD pattern is utilized to analyze different processing conditions affecting on the formation of quasi-2D perovskite crystal phase and crystallinity, as shown in Figure 1(k) and Figure S3. The DMF RT film shows weak diffraction peak at $13.95^{\circ}$ and $27.9^{\circ}$, indicating its poor crystallinity. The diffraction peak intensity of DMSO RT 
film is stronger than that of DMF RT film distinctly. However, accordingly to the enlarged XRD pattern shown in Figure 1(k), the diffraction peaks are located at $6.7^{\circ}$, $8.9^{\circ}$ and $10.2^{\circ}$, which should be assigned to different lower $n$-number phases $(n=2 \&$ $n=3)^{49}$. As it is expected, the film with $10 \% \mathrm{~K}^{+}$additive exhibits two dominant peaks at $14^{\circ}$ and $28^{\circ}$ that correspond to (111) and (222) lattice planes of quasi-2D perovskite, respectively, and the diffraction peak intensity is dramatically enhanced, which is consistent with hot-cast technique. Meanwhile, the enlarged XRD pattern shows no extra diffraction peaks at small angle, indicating that the phase distribution becomes narrow. When doping different $\mathrm{K}^{+}$concentration, all the diffraction peak intensities are enhanced compared with that of reference one. However, especially doped $20 \% \mathrm{~K}^{+}$, the film exhibits weak series of crystal diffraction of $(0 \mathrm{k} 0)$ lattice plane of quasi-2D perovskite, which manifests that the superfluous $\mathrm{K}^{+}$prompts the formation of lower $n$ number phases. Besides, there is no shift for the diffraction peak of (111), as shown in Figure S3 (b), indicating that $\mathrm{K}^{+}$incorporation could not change the crystal structure. Figure 1(I) shows the full-width at half-maximum (FWHM) and crystallite size of quasi-2D perovskite films for the (111) lattice plane with different processing conditions, the corresponding parameters are plotted in Table S1. The FWHM of DMSO RT, hot-cast technique and doped $10 \% \mathrm{~K}^{+}$films all decrease from $0.66^{\circ}, 0.17^{\circ}$ to $0.16^{\circ}$, respectively, indicating the film with $\mathrm{K}^{+}$displays superior crystallinity compared with reference one. In order to further investigate the crystal quality, we calculate the crystallite size of specific lattice plane according to Scherrer formula, ${ }^{51}$ the detailed calculation of average crystallite size is discussed in Supporting 
Information ST2. Regarding to DMSO RT film, crystallite size increases from $12 \mathrm{~nm}$ (DMF RT) to $35 \mathrm{~nm}$, and the crystallite size of hot-cast film even reaches to $48 \mathrm{~nm}$. When doped $10 \% \mathrm{~K}^{+}$, the crystallite size is as large as $51 \mathrm{~nm}$. The larger crystallite size decreases the formation of grain boundaries, which can reduce non-radiative recombination and is beneficial to the solar cell performance.

To further investigate the vertical oriented formation mechanism for KBAMA film, we particularly investigate the crystallinity process of quasi-2D perovskite films. KI aggregates, which show much lower solubility in DMF than either MAI-PbI 2 or BAI$\mathrm{PbI}_{2}$ colloids, would likely generate early precipitate out of the solution during spincoating process. In this case, we suspect that any generated KI aggregates would act as crystal nucleus at the air-liquid interface in the early stage of spin-coating, leading to the further heterogeneous crystallization with vertical distribution of perovskite materials. Similar mechanism of nucleating at air-liquid interface has already been conducted in the former work. ${ }^{43}$ Therefore, we speculate that the crystallization for doped $10 \%$ KBAMA film has already incurred along vertical crystallization during the spin-coating process, which is as similar as hot-cast process. To verify the above speculation, the XRD patterns for quasi-2D perovskite films before and after the annealing are shown in Figure S4. The 0\% KBAMA film displays different XRD spectra before and after the thermal annealing. Before the annealing, there are intermediate phases in the film where crystallization process has not finished yet. However, there is no difference between the XRD spectra of doped 10\% KBAMA film before and after thermal annealing process, which could also indicate that the film has 
already finished the crystallization. Doped 10\% KBAMA film thickness has nearly no change before and after the annealing, as shown in Table S3. On the contrary, there is large film thickness variation of $30-60 \mathrm{~nm}$ for $0 \%$ KBAMA film before and after the annealing. This observation is consistent with the above XRD measurement.

Besides, we observed the crystallization dynamics and compositional control mechanism of doped $0 \%$ and $10 \% \mathrm{~K}^{+}$quasi-2D perovskite films during spin-coating process by in-situ synchrotron-based GIXRD characterization. The testing schematic diagram is shown in Figure 2(a), (b). The in-situ GIXRD signals for one-minute formation process of $0 \%$ and $10 \% \mathrm{~K}^{+}$quasi-2D perovskite films are recorded by a supporting video (SV) of SV01 and SV02, respectively. From Figure 2(c)-(g), diffraction signals of perovskite grains of doped 0\% KBAMA film appear at approximately $12 \mathrm{~s}$, and signals for low $n$-number intermediate phases could be clearly observed after $20 \mathrm{~s}$. When further increasing spin-coating process time, the signals of crystal grains enhanced with random orientation, which is consistent with previous results. By comparison for the doped 10\% KBAMA film, as shown in Figure 2(h)-(l), the crystallization signals appeared at $15 \mathrm{~s}$ and enhanced significantly without any intermediate phases when further increasing spinning coating time. And the vertical orientation has formed even without annealing process. Figure S5 shows the azimuthally integrated intensity profiles for these two films derived from Figure 2(c)(g) $\left(0 \% \mathrm{~K}^{+}\right)$and Figure 2(h)-(l) $\left(10 \% \mathrm{~K}^{+}\right)$, respectively. The diffraction peak (111) of perovskite films becomes very sharp after $\sim 15 \mathrm{~s}$ spin-coasting elapsed process. The sharp and strong diffraction peak (111) indicates that the film crystallization incurs in 
this stage. And the crystallization process would finish during spin-coating method. Besides, the corresponding radially integrated intensity of the diffraction rings at $q \approx$ $10 \mathrm{~nm}^{-1}$ at different five time points $(0,12,20,40,60 \mathrm{~s})$ for $0 \% \mathrm{~K}^{+}$film and $(0,15,20$, $40,60 \mathrm{~s}$ ) for $10 \% \mathrm{~K}^{+}$film are showed in Figure S6. The film with $0 \% \mathrm{~K}^{+}$shows several small peaks at different azimuth angles, which could not be found in $10 \% \mathrm{~K}^{+}$film, indicating that the film with $0 \% \mathrm{~K}^{+}$crystallizes with random orientations. The results further verify the above hypothesis that the incorporation of $\mathrm{K}^{+}$can directly convert to vertical oriented quasi-2D perovskite phase. Besides, according to the perovskite diffraction peaks (111) for $0 \% \mathrm{~K}^{+}$and $10 \% \mathrm{~K}^{+}$films with different spin-coating time in Figure S7(a)-(b), we could clearly find that the intensity of diffraction peak (111) of film with $0 \% \mathrm{~K}^{+}$reaches its saturation point at approximately $20 \mathrm{~s}$. However, the intensity of $10 \% \mathrm{~K}^{+}$film reaches similar value at $20 \mathrm{~s}$ compared with $0 \% \mathrm{~K}^{+}$film, and further increases till the end of spin-coating process. This result indicates the better crystallinity of $\mathrm{K}^{+}$doped film. Figure S7(c)-(e) plot the diffraction peak (111) position, intensity (peak area) and FWHM from films with different spin-coating time, which reveal the more symmetric crystallization structure and improved crystallinity for $\mathrm{K}^{+}$ incorporated film.

To probe the phase distribution and light absorption property of quasi-2D perovskite films with different processing conditions, ultraviolet visible (UV-vis) absorption and photoluminescence (PL) characterizations are conducted, as shown in Figure 3 and Figure S8, S9. According to the UV-vis absorption spectra, it is observed that the films display multiple phases containing different $n$ value although all precursor solutions are 
prepared according to formula of $\mathrm{BA}_{2} \mathrm{MA}_{3} \mathrm{~Pb}_{4} \mathrm{I}_{13}$. When DMF is used as solvent, the absorption peaks representing $2 \mathrm{D}$ phases are almost invisible due to the prominent $3 \mathrm{D}$ perovskite phase. The slowing crystallinity prompts to the formation of different $2 \mathrm{D}$ phases when adopting DMSO as solvent. For the quasi-2D perovskite processed with hot-cast technique, excitonic peaks at $\sim 570 \mathrm{~nm}, \sim 606 \mathrm{~nm}, \sim 642 \mathrm{~nm}$ are observed, corresponding to different quasi-2D phases $(n=2,3,4)$. Once $\mathrm{K}^{+}$is incorporated, the excitonic peaks representing $n=2,3,4$ remain un-shifted. Dramatically, the absorption peak for $n=3$ are prominently enhanced while other peaks are weakened. In other hand, the incorporation of $\mathrm{K}^{+}$can enable the formation of quasi-2D perovskite phases, being as similar as the signal observed from the 2D perovskite film processed with hot-cast technique and DMSO solvent.

The PL peak position can be different in quasi-2D perovskite when pumping film along two different directions either from the front side (surface of the perovskite film) or the rear one (substrate) if quasi-2D perovskite containing large phase distribution ${ }^{52}$. PL measurement is conducted to further probe phase distribution of quasi-2D perovskite films fabricated under different processing conditions, as shown in Figure 3(c), (d) and Figure S6. When probed from the front side, the films with different processing conditions all display one peak. However, the PL peaks of DMSO RT (770 nm), hotcast technique $(777 \mathrm{~nm})$ and doped $10 \% \mathrm{~K}^{+}$films $(777 \mathrm{~nm})$ all shift to longer wavelength approaching to $778 \mathrm{~nm}$, being consistent with the peak position of 3D perovskite. When probed from the back side, the film processed with hot-cast technique exhibits obvious extra peaks at $577 \mathrm{~nm}, 617 \mathrm{~nm}, 652 \mathrm{~nm}$, as shown enlarged PL peak 
in Figure 3(d), representing different low $n$-number phases $(n=2,3,4)$, which is consistent with the absorption exciton peak. In contrast there is still one PL peak for doped $10 \% \mathrm{~K}^{+}$film when pumping rear side. It can be safely concluded that the doped $10 \% \mathrm{~K}^{+}$film reduces the distribution of different phases according $\mathrm{UV}$-vis absorption and PL spectra measurement. When further increasing the $\mathrm{K}^{+}$concentration to $20 \%$, the low $n$-number phases $(n<4)$ increase again according to UV-vis absorption (Figure S5) and PL spectra measurement (Figure S6), which is consistent with XRD result (Figure 1k). In addition, the PL intensity of doped $10 \% \mathrm{~K}^{+}$film reaches the peak value. Further increasing the concentration of $\mathrm{K}^{+}$results in deteriorating PL, which is likely correlated with the existence of extra multiple $2 \mathrm{D}$ phases. ${ }^{53}$ Beside the difference of PL intensity, the main PL peak begins to red shift when doped $5 \% \mathrm{~K}^{+}$, and the peak position remains no obvious shift when further increasing the concentration of $\mathrm{K}^{+}$. Combined with the PL spectra of the film fabricated with different processing conditions, it is inferred that the low- $n$ phase is favored at the bottom and high- $n$ phase is inclined at the top, the schematic diagram is shown in Figure 3(b).

The suitable orientation and organized phase in quasi-2D perovskite are both very critical for optoelectronic device application ${ }^{28-29}$. LED depends on the quantum well confinement existed between multiple different vertical oriented phases, which is favorable to efficient electron-hole recombination and carrier transport. Similarly, the quasi-2D perovskite solar cell performance is affected by multiple factors, mainly including phase distribution, vertical orientation, crystallization and grain size, which can suppress the carrier recombination and accelerate charge transport. To investigate 
how crystal orientation and phase distribution impact the performance of quasi-2D perovskite solar cells, we prepare conventional devices with different processing conditions, the device structure is shown in Figure S10(a). The structure is fluorinedoped tin oxide glass (FTO) $/ \mathrm{TiO}_{\mathrm{x}} /$ perovskite/2,2' $, 7,7^{\prime}-$ Tetrakis[N,N-di(4methoxyphenyl)amino]-9,9'-spirobifluorene (Spiro-OMeTAD) /Ag. Figure 4(a) displays the current density-voltage $(J-V)$ curves of quasi-2D perovskite solar cells with different processing conditions under simulated air mass (AM) $1.5 \mathrm{G}$ one sun illumination. The electrical output characteristics of all the devices are summarized in Table 1 and Table S2. The PCE of KBAMA devices gradually increases with increasing $\mathrm{K}^{+}$concentration. The PCE of $10 \%$ KBAMA device reaches a peak value of $11.3 \%$ with the open-circuit voltage $\left(V_{o c}\right)$ of $1.00 \mathrm{~V}$, short current density $\left(J_{s c}\right)$ of 18.9 $\mathrm{mA} \mathrm{cm}{ }^{-2}$, and fill factor $(F F)$ of $52 \%$. The PCE is obviously superior to BAMA device, especially the $J_{s c}$ and $F F$ values, which is correlated with the vertical orientation and organized phase. The hot-cast technique fabricated device with PCE of $9.82 \%$ is lower than that of $10 \%$ KBAMA device, but the $J_{s c}$ is slightly higher and $F F$ is poorer because thickness of $10 \%$ KBAMA film $(\sim 180 \mathrm{~nm})$ is thinner than that of hot-cast technique $(\sim 250 \mathrm{~nm})$. With further increasing $\mathrm{K}^{+}$concentration $(>10 \%)$, PCE begins reduced (Figure S11), which is consistent with the above poor film morphology and inferior UV-vis and PL characteristics. Particularly doped $20 \% \mathrm{~K}^{+}$perovskite solar cell, the PCE reduces from $11.3 \%$ to $8.2 \%$. The hot-cast technique film can achieve the highly crystallization and vertical orientation ${ }^{29,54}$, however, the poor controllability retards further development due to difficult manipulation the whole film fabrication process. 
To investigate the $10 \%$ KBAMA device repeatability compared with the hot-cast technique, the distribution of 25 devices with hot-cast technique and doped $10 \% \mathrm{~K}^{+}$ KBAMA one is plotted in Figure 4(b). The PCE of BAMA devices with hot-cast technique distributes between $6.6 \%$ and $9.8 \%$, however, the $10 \%$ KBAMA devices distribute between $10.5 \%$ and $11.3 \%$, which indicates that the latter device performance not only surpasses the former, but also has decent reproducibility. Besides, according to previous report, ${ }^{40}$ vertical oriented quasi-2D perovskite film can not only benefit charge transport but also improve the moisture stability. We also investigated the longterm stability of un-encapsulated $10 \% \mathrm{KBAMA}$ and $3 \mathrm{D} \mathrm{MAPbI}_{3}$ devices stored in air under dark for $1000 \mathrm{~h}$ in $40 \% \pm 10 \%$ relative humidity, details are shown in Supporting

\section{Information ST3.}

The effect on the performance of quasi-2D perovskite devices is not only ascribed to vertical oriented crystallinity, but also correlated with the trap states in perovskite films. ${ }^{55}$ To further evaluate the trap densities of doped $0 \% \mathrm{~K}^{+}$and $10 \% \mathrm{~K}^{+}$quasi-2D perovskite films, space-charge-limited-current (SCLC) method by fabricating electrononly devices was conducted. ${ }^{56-57}$ Figure S13 shows dark $J-V$ curves of electron-only devices with different perovskite films. The primary linear region represents the Ohmic contact when the bias voltage is low enough. The intermediate region can be regarded as trap filling process when increasing the current injection. Further increasing the bias, the trap states are constantly filled until reaching the trap-filling limit voltage $\left(V_{\mathrm{TFL}}\right)$. The formula detail can be expressed in Supporting Information ST4. Therefore, lower $V_{\mathrm{TFL}}$ reflects the low concentration of defect states. The DMF RT quasi-2D BAMA 
device shows higher $V_{\mathrm{TFL}}(0.88 \mathrm{~V})$, however, upon doped $10 \% \mathrm{~K}^{+}$, the $V_{\mathrm{TFL}}$ value is obviously lower to $0.26 \mathrm{~V}$. Therefore, the corresponding calculated trap density for KBAMA film reduced from $9.6 \times 10^{16} \mathrm{~cm}^{-3}$ (BAMA) to $2.8 \times 10^{16} \mathrm{~cm}^{-3}$. The calculated trap density for KBAMA film reduced from $9.6 \times 10^{16} \mathrm{~cm}^{-3}$ (BAMA) to $2.8 \times 10^{16} \mathrm{~cm}^{-3}$. The excellent crystallinity and vertical orientation for quasi-2D 10\% KBAMA perovskite dramatically suppresses the defect states.

In summary, we have developed a simple method to achieve the desirable vertical oriented quasi-2D perovskite film for high efficiency and stable solar cell via incorporation of $\mathrm{K}^{+}$. Compared with the reference film, it has been found that the $\mathrm{K}^{+}$ doping perovskite precursor wet film converts directly to desirable $2 \mathrm{D}$ phase with excellent crystallinity along vertical orientation even in spin-coating process monitored by real time in-situ synchrotron-based GIXRD. Meanwhile, the vertical oriented phase could occupy the majority of crystallized film with the incorporation of $\mathrm{K}^{+}$. The easier crystallization step at air-liquid interface can well interpret the preferred oriented crystallization process. Accordingly, the optimal 10\% KBAMA device achieves the PCE of $11.3 \%$ and the un-encapsulated device can maintain $90 \%$ of initial performance after $1000 \mathrm{~h}$ in $40 \% \pm 10 \%$ relative humidity at room temperature. In addition, the devices simultaneously show high repeatability. Our work provides one new method for the orientation regulation of quasi-2D perovskite apart from the popular hot-cast technique.

\section{Supporting Information}

Supporting Information is available from the ACS publication or from the authors. The 
videos of in-situ GIXRD characterization for perovskite films could also be found in Supporting Information.

\section{Acknowledgements}

This work was supported by the National Key Research and Development Program of China (2016YFA0202402, 2017YFA0403400), the National Natural Science Foundation of China (61974098, 61674108), Jiangsu High Educational Natural Science Foundation (18KJA430012), the Priority Academic Program Development of Jiangsu Higher Education Institutions, the 111 program and Collaborative Innovation Center of Suzhou Nano Science and Technology (NANO-CIC), Programme Decouverte Chine 2019 (project 43619UM) and the PROCES project (ANR-17-CEO5-0028).

\section{Conflict of Interest}

The authors declare no conflict of interest.

\section{References}

1. Green, M. A.; Ho-Baillie, A.; Snaith, H. J., The emergence of perovskite solar cells. Nat. Photonics 2014, 8, 506.

2. Lin, Z.; Park, N.-G.; Li, G., Themed issue on perovskite solar cells. J. Mater. Chem. A 2015, 3 (17), 8924-8925.

3. Yin, W.-J.; Shi, T.; Yan, Y., Unique Properties of Halide Perovskites as Possible Origins of the Superior Solar Cell Performance. Adv. Mater. 2014, 26 (27), 4653-4658.

4. Stranks, S. D.; Eperon, G. E.; Grancini, G.; Menelaou, C.; Alcocer, M. J. P.; Leijtens, T.; Herz, L. M.; Petrozza, A.; Snaith, H. J., Electron-Hole Diffusion Lengths Exceeding 1 Micrometer in an Organometal Trihalide Perovskite Absorber. Science 
2013, 342 (6156), 341.

5. Pellet, N.; Gao, P.; Gregori, G.; Yang, T.-Y.; Nazeeruddin, M. K.; Maier, J.; Grätzel, M., Mixed-Organic-Cation Perovskite Photovoltaics for Enhanced Solar-Light Harvesting. Angew. Chem. Int. Ed. 2014, 53 (12), 3151-3157.

6. Park, N.-G., Perovskite solar cells: an emerging photovoltaic technology. Mater. Today 2015, 18 (2), 65-72.

7. Kim, Y.-H.; Cho, H.; Heo, J. H.; Kim, T.-S.; Myoung, N.; Lee, C.-L.; Im, S. H.; Lee, T.-W., Multicolored Organic/Inorganic Hybrid Perovskite Light-Emitting Diodes. Adv. Mater. 2015, 27 (7), 1248-1254.

8. Wang, N.; Cheng, L.; Ge, R.; Zhang, S.; Miao, Y.; Zou, W.; Yi, C.; Sun, Y.; Cao, Y.; Yang, R.; Wei, Y.; Guo, Q.; Ke, Y.; Yu, M.; Jin, Y.; Liu, Y.; Ding, Q.; Di, D.; Yang, L.; Xing, G.; Tian, H.; Jin, C.; Gao, F.; Friend, R. H.; Wang, J.; Huang, W., Perovskite light-emitting diodes based on solution-processed self-organized multiple quantum wells. Nat. Photonics 2016, 10, 699.

9. Lin, Q.; Armin, A.; Lyons, D. M.; Burn, P. L.; Meredith, P., Low Noise, IR-Blind Organohalide Perovskite Photodiodes for Visible Light Detection and Imaging. $A d v$. Mater. 2015, 27 (12), 2060-2064.

10. Saidaminov, M. I.; Adinolfi, V.; Comin, R.; Abdelhady, A. L.; Peng, W.; Dursun, I.; Yuan, M.; Hoogland, S.; Sargent, E. H.; Bakr, O. M., Planar-integrated singlecrystalline perovskite photodetectors. Nat. Commun. 2015, 6, 8724.

11. Green, M. A.; Hishikawa, Y.; Dunlop, E. D.; Levi, D. H.; Hohl-Ebinger, J.; HoBaillie, A. W. Y., Solar cell efficiency tables (version 52). 
(https://onlinelibrary.wiley.com/doi/abs/10.1002/pip.3040) Progress in Photovoltaics: Research and Applications 2018, 26 (7), 427-436.

12. Niu, G.; Guo, X.; Wang, L., Review of recent progress in chemical stability of perovskite solar cells. J. Mater. Chem. A 2015, 3 (17), 8970-8980.

13. Wang, D.; Wright, M.; Elumalai, N. K.; Uddin, A., Stability of perovskite solar cells. Sol. Energy Mater. Sol. Cells 2016, 147, 255-275.

14. Tan, H.; Jain, A.; Voznyy, O.; Lan, X.; García de Arquer, F. P.; Fan, J. Z.; QuinteroBermudez, R.; Yuan, M.; Zhang, B.; Zhao, Y.; Fan, F.; Li, P.; Quan, L. N.; Zhao, Y.; Lu, Z.-H.; Yang, Z.; Hoogland, S.; Sargent, E. H., Efficient and stable solution-processed planar perovskite solar cells via contact passivation. Science 2017, 355 (6326), 722.

15. Bella, F.; Griffini, G.; Correa-Baena, J.-P.; Saracco, G.; Grätzel, M.; Hagfeldt, A.; Turri, S.; Gerbaldi, C., Improving efficiency and stability of perovskite solar cells with photocurable fluoropolymers. Science 2016, 354 (6309), 203.

16. Zhou, H.; Chen, Q.; Li, G.; Luo, S.; Song, T.-b.; Duan, H.-S.; Hong, Z.; You, J.; Liu, Y.; Yang, Y., Interface engineering of highly efficient perovskite solar cells. Science 2014, 345 (6196), 542.

17. Li, W.; Zhang, W.; Van Reenen, S.; Sutton, R. J.; Fan, J.; Haghighirad, A. A.; Johnston, M. B.; Wang, L.; Snaith, H. J., Enhanced UV-light stability of planar heterojunction perovskite solar cells with caesium bromide interface modification. Energy Environ. Sci. 2016, 9 (2), 490-498.

18. Zuo, L.; Gu, Z.; Ye, T.; Fu, W.; Wu, G.; Li, H.; Chen, H., Enhanced Photovoltaic Performance of $\mathrm{CH} 3 \mathrm{NH} 3 \mathrm{PbI} 3$ Perovskite Solar Cells through Interfacial Engineering 
Using Self-Assembling Monolayer. J. Am. Chem. Soc. 2015, 137 (7), 2674-2679.

19. Li, Y.; Zhao, Y.; Chen, Q.; Yang, Y.; Liu, Y.; Hong, Z.; Liu, Z.; Hsieh, Y.-T.; Meng, L.; Li, Y.; Yang, Y., Multifunctional Fullerene Derivative for Interface Engineering in Perovskite Solar Cells. J. Am. Chem. Soc. 2015, 137 (49), 15540-15547.

20. Saliba, M.; Matsui, T.; Seo, J.-Y.; Domanski, K.; Correa-Baena, J.-P.; Nazeeruddin, M. K.; Zakeeruddin, S. M.; Tress, W.; Abate, A.; Hagfeldt, A.; Grätzel, M., Cesiumcontaining triple cation perovskite solar cells: improved stability, reproducibility and high efficiency. Energy Environ. Sci. 2016, 9 (6), 1989-1997.

21. Beal, R. E.; Slotcavage, D. J.; Leijtens, T.; Bowring, A. R.; Belisle, R. A.; Nguyen, W. H.; Burkhard, G. F.; Hoke, E. T.; McGehee, M. D., Cesium Lead Halide Perovskites with Improved Stability for Tandem Solar Cells. J Phys Chem Lett 2016, 7 (5), 746751.

22. Tiep, N. H.; Ku, Z.; Fan, H. J., Recent Advances in Improving the Stability of Perovskite Solar Cells. Adv. Energy Mater. 2016, 6 (3), 1501420.

23. Gao, P.; Bin Mohd Yusoff, A. R.; Nazeeruddin, M. K., Dimensionality engineering of hybrid halide perovskite light absorbers. Nat. Commun. 2018, 9 (1), 5028.

24. Bai, Y.; Xiao, S.; Hu, C.; Zhang, T.; Meng, X.; Lin, H.; Yang, Y.; Yang, S., Dimensional Engineering of a Graded 3D-2D Halide Perovskite Interface Enables Ultrahigh Voc Enhanced Stability in the p-i-n Photovoltaics. Adv. Energy Mater. 2017, $7(20), 1701038$.

25. Lim, J. W.; Wang, H.; Choi, C. H.; Kwon, H.; Quan, L. N.; Park, W.-T.; Noh, Y.Y.; Kim, D. H., Self-powered reduced-dimensionality perovskite photodiodes with 
controlled crystalline phase and improved stability. Nano Energy 2019, 57, 761-770.

26. Zhang, Q.; Chu, L.; Zhou, F.; Ji, W.; Eda, G., Excitonic Properties of Chemically Synthesized 2D Organic-Inorganic Hybrid Perovskite Nanosheets. Adv. Mater. 2018, $30(18), 1704055$.

27. Blancon, J. C.; Stier, A. V.; Tsai, H.; Nie, W.; Stoumpos, C. C.; Traoré, B.; Pedesseau, L.; Kepenekian, M.; Katsutani, F.; Noe, G. T.; Kono, J.; Tretiak, S.; Crooker, S. A.; Katan, C.; Kanatzidis, M. G.; Crochet, J. J.; Even, J.; Mohite, A. D., Scaling law for excitons in 2D perovskite quantum wells. Nat. Commun. 2018, 9 (1), 2254.

28. Zhang, X.; Wu, G.; Fu, W.; Qin, M.; Yang, W.; Yan, J.; Zhang, Z.; Lu, X.; Chen, H., Orientation Regulation of Phenylethylammonium Cation Based 2D Perovskite Solar Cell with Efficiency Higher Than 11\%. Adv. Energy Mater. 2018, 8(14), 1702498. 29. Tsai, H.; Nie, W.; Blancon, J.-C.; Stoumpos, C. C.; Asadpour, R.; Harutyunyan, B.; Neukirch, A. J.; Verduzco, R.; Crochet, J. J.; Tretiak, S.; Pedesseau, L.; Even, J.; Alam, M. A.; Gupta, G.; Lou, J.; Ajayan, P. M.; Bedzyk, M. J.; Kanatzidis, M. G.; Mohite, A. D., High-efficiency two-dimensional Ruddlesden-Popper perovskite solar cells. Nature 2016, 536, 312 .

30. Zhang, T.; Dar, M. I.; Li, G.; Xu, F.; Guo, N.; Grätzel, M.; Zhao, Y., Bication lead iodide $2 \mathrm{D}$ perovskite component to stabilize inorganic $\alpha-\mathrm{CsPbI} 3$ perovskite phase for high-efficiency solar cells. Sci. Adv. 2017, 3 (9), e1700841.

31. Chen, P.; Bai, Y.; Wang, S.; Lyu, M.; Yun, J.-H.; Wang, L., In Situ Growth of 2D Perovskite Capping Layer for Stable and Efficient Perovskite Solar Cells. Adv. Funct. Mater. 2018, 28 (17), 1706923. 
32. Li, G.; Zhang, T.; Guo, N.; Xu, F.; Qian, X.; Zhao, Y., Ion-Exchange-Induced 2D3D Conversion of HMA1-xFAxPbI3Cl Perovskite into a High-Quality MA1-xFAxPbI3 Perovskite. Angew. Chem. Int. Ed. 2016, 55 (43), 13460-13464.

33. Grancini, G.; Roldán-Carmona, C.; Zimmermann, I.; Mosconi, E.; Lee, X.; Martineau, D.; Narbey, S.; Oswald, F.; De Angelis, F.; Graetzel, M.; Nazeeruddin, M. K., One-Year stable perovskite solar cells by 2D/3D interface engineering. Nat. Commun. 2017, 8, 15684.

34. Wang, Z.; Lin, Q.; Chmiel, F. P.; Sakai, N.; Herz, L. M.; Snaith, H. J., Efficient ambient-air-stable solar cells with 2D-3D heterostructured butylammonium-caesiumformamidinium lead halide perovskites. Nat. Energy 2017, 2, 17135.

35. Ye, T.; Bruno, A.; Han, G.; Koh, T. M.; Li, J.; Jamaludin, N. F.; Soci, C.; Mhaisalkar, S. G.; Leong, W. L., Efficient and Ambient-Air-Stable Solar Cell with Highly Oriented 2D@3D Perovskites. Adv. Funct. Mater. 2018, 28 (30), 1801654.

36. Wu, C.; Wu, T.; Yang, Y.; McLeod, J. A.; Wang, Y.; Zou, Y.; Zhai, T.; Li, J.; Ban, M.; Song, T.; Gao, X.; Duhm, S.; Sirringhaus, H.; Sun, B., Alternative Type TwoDimensional-Three-Dimensional Lead Halide Perovskite with Inorganic Sodium Ions as a Spacer for High-Performance Light-Emitting Diodes. ACS Nano 2019, 13 (2), $1645-1654$.

37. Chen, Y.; Yu, S.; Sun, Y.; Liang, Z., Phase Engineering in Quasi-2D RuddlesdenPopper Perovskites. J Phys Chem Lett 2018, 9 (10), 2627-2631.

38. Tsai, H.; Nie, W.; Blancon, J.-C.; Stoumpos, C. C.; Soe, C. M. M.; Yoo, J.; Crochet, J.; Tretiak, S.; Even, J.; Sadhanala, A.; Azzellino, G.; Brenes, R.; Ajayan, P. M.; Bulović, 
V.; Stranks, S. D.; Friend, R. H.; Kanatzidis, M. G.; Mohite, A. D., Stable LightEmitting Diodes Using Phase-Pure Ruddlesden-Popper Layered Perovskites. $A d v$. Mater. 2018, 30 (6), 1704217.

39. Zhang, X.; Munir, R.; Xu, Z.; Liu, Y.; Tsai, H.; Nie, W.; Li, J.; Niu, T.; Smilgies, D.-M.; Kanatzidis, M. G.; Mohite, A. D.; Zhao, K.; Amassian, A.; Liu, S., Phase Transition Control for High Performance Ruddlesden-Popper Perovskite Solar Cells. Adv. Mater. 2018, 30 (21), 1707166.

40. Soe, C. M. M.; Nie, W.; Stoumpos, C. C.; Tsai, H.; Blancon, J.-C.; Liu, F.; Even, J.; Marks, T. J.; Mohite, A. D.; Kanatzidis, M. G., Understanding Film Formation Morphology and Orientation in High Member 2D Ruddlesden-Popper Perovskites for High-Efficiency Solar Cells. Adv. Energy Mater. 2018, 8 (1), 1700979.

41. Williams, O. F.; Guo, Z.; Hu, J.; Yan, L.; You, W.; Moran, A. M., Energy transfer mechanisms in layered 2D perovskites. J Chem Phys. 2018, 148 (13), 134706.

42. Milot, R. L.; Sutton, R. J.; Eperon, G. E.; Haghighirad, A. A.; Martinez Hardigree, J.; Miranda, L.; Snaith, H. J.; Johnston, M. B.; Herz, L. M., Charge-Carrier Dynamics in 2D Hybrid Metal-Halide Perovskites. Nano Lett. 2016, 16 (11), 7001-7007.

43. Chen, A. Z.; Shiu, M.; Ma, J. H.; Alpert, M. R.; Zhang, D.; Foley, B. J.; Smilgies, D.-M.; Lee, S.-H.; Choi, J. J., Origin of vertical orientation in two-dimensional metal halide perovskites and its effect on photovoltaic performance. Nat. Commun. 2018, 9 (1), 1336 .

44. Fu, W.; Wang, J.; Zuo, L.; Gao, K.; Liu, F.; Ginger, D. S.; Jen, A. K. Y., TwoDimensional Perovskite Solar Cells with 14.1\% Power Conversion Efficiency and 0.68\% 
External Radiative Efficiency. ACS Energy Lett. 2018, 3 (9), 2086-2093.

45. Lai, H.; Kan, B.; Liu, T.; Zheng, N.; Xie, Z.; Zhou, T.; Wan, X.; Zhang, X.; Liu, Y.;

Chen, Y., Two-Dimensional Ruddlesden-Popper Perovskite with Nanorod-like Morphology for Solar Cells with Efficiency Exceeding 15\%. J. Am. Chem. Soc. 2018, 140 (37), 11639-11646.

46. Kuai, L.; Wang, Y.; Zhang, Z.; Yang, Y.; Qin, Y.; Wu, T.; Li, Y.; Li, Y.; Song, T.; Gao, X.; Wang, L.; Sun, B., Passivating Crystal Boundaries with Potassium-Rich Phase in Organic Halide Perovskite. Sol. RRL 2019, 3 (5), 1900053.

47. Qiu, J.; Zheng, Y.; Xia, Y.; Chao, L.; Chen, Y.; Huang, W., Rapid Crystallization for Efficient 2D Ruddlesden-Popper (2DRP) Perovskite Solar Cells. Adv. Funct. Mater. 2018, 1806831.

48. Lv, M.; Dong, X.; Fang, X.; Lin, B.; Zhang, S.; Ding, J.; Yuan, N., A promising alternative solvent of perovskite to induce rapid crystallization for high-efficiency photovoltaic devices. $R S C A d v$. 2015, 5 (26), 20521-20529.

49. Quan, L. N.; Yuan, M.; Comin, R.; Voznyy, O.; Beauregard, E. M.; Hoogland, S.; Buin, A.; Kirmani, A. R.; Zhao, K.; Amassian, A.; Kim, D. H.; Sargent, E. H., LigandStabilized Reduced-Dimensionality Perovskites. J. Am. Chem. Soc. 2016, 138 (8), 2649-2655.

50. Mei, A.; Li, X.; Liu, L.; Ku, Z.; Liu, T.; Rong, Y.; Xu, M.; Hu, M.; Chen, J.; Yang, Y.; Grätzel, M.; Han, H., A hole-conductor-free, fully printable mesoscopic perovskite solar cell with high stability. Science 2014, 345 (6194), 295.

51. Patterson, A. L., The Scherrer Formula for X-Ray Particle Size Determination. 
Phys. Rev. 1939, 56 (10), 978-982.

52. Liu, J.; Leng, J.; Wu, K.; Zhang, J.; Jin, S., Observation of Internal Photoinduced Electron and Hole Separation in Hybrid Two-Dimentional Perovskite Films. J. Am. Chem. Soc. 2017, 139 (4), 1432-1435.

53. Fu, Y.; Zheng, W.; Wang, X.; Hautzinger, M. P.; Pan, D.; Dang, L.; Wright, J. C.; Pan, A.; Jin, S., Multicolor Heterostructures of Two-Dimensional Layered Halide Perovskites that Show Interlayer Energy Transfer. J. Am. Chem. Soc. 2018, 140 (46), 15675-15683.

54. Quintero-Bermudez, R.; Gold-Parker, A.; Proppe, A. H.; Munir, R.; Yang, Z.; Kelley, S. O.; Amassian, A.; Toney, M. F.; Sargent, E. H., Compositional and orientational control in metal halide perovskites of reduced dimensionality. Nat. Mater. 2018, 17 (10), 900-907.

55. Zhang, X.; Ren, X.; Liu, B.; Munir, R.; Zhu, X.; Yang, D.; Li, J.; Liu, Y.; Smilgies, D.-M.; Li, R.; Yang, Z.; Niu, T.; Wang, X.; Amassian, A.; Zhao, K.; Liu, S., Stable high efficiency two-dimensional perovskite solar cells via cesium doping. Energy Environ. Sci. 2017, 10 (10), 2095-2102.

56. Mihailetchi, V. D.; Wildeman, J.; Blom, P. W. M., Space-Charge Limited Photocurrent. Phys. Rev. Lett. 2005, 94 (12), 126602.

57. Lampert, M. A., Simplified Theory of Space-Charge-Limited Currents in an Insulator with Traps. Phys. Rev. 1956, 103 (6), 1648-1656. 
Figures and Captions
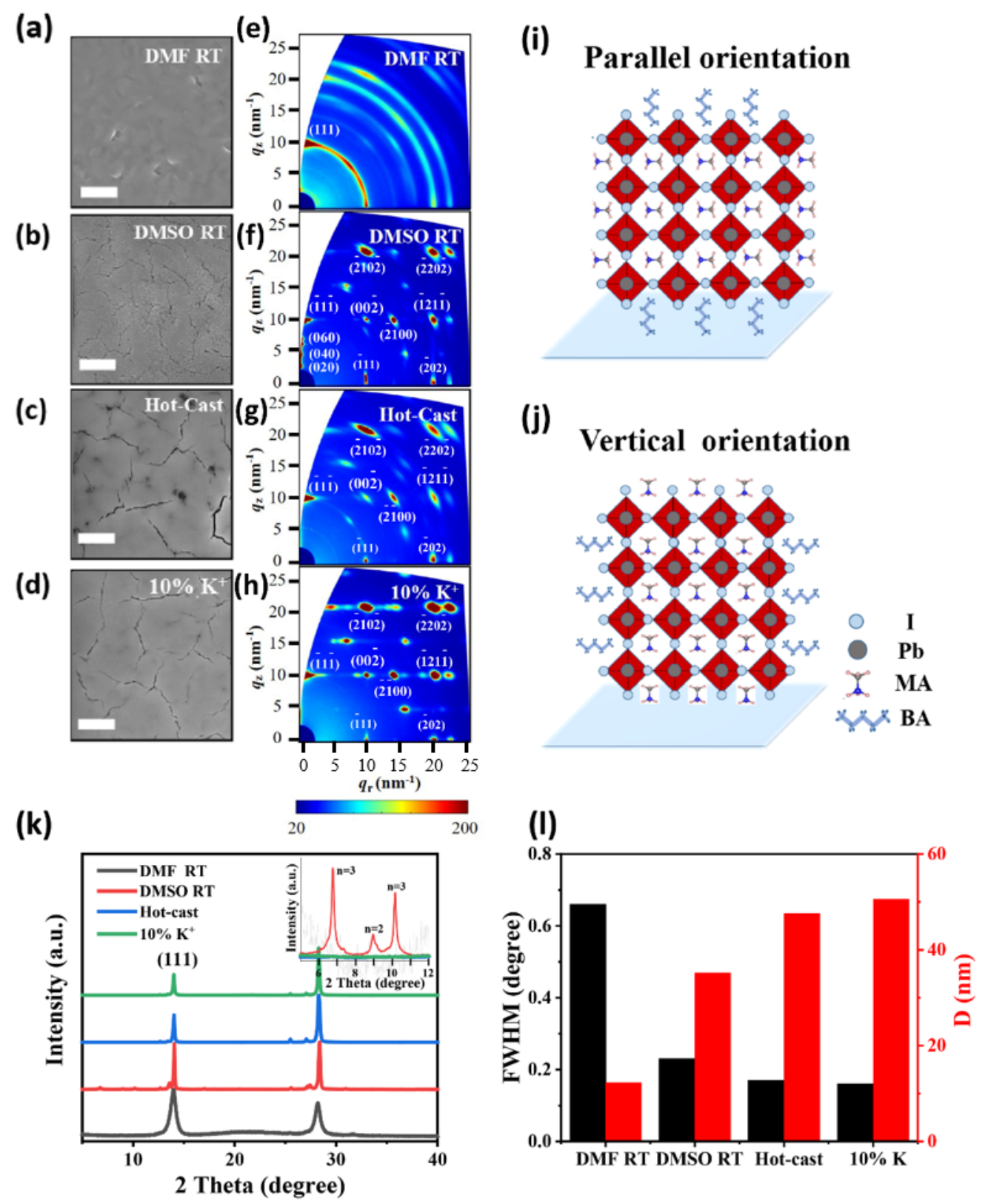

Figure 1 SEM images of quasi-2D perovskite thin films with different processing conditions in a) DMF RT, b) DMSO RT, c) Hot-cast technique and d) doped $10 \% \mathrm{~K}^{+}$. All the scale bars are $1 \mu \mathrm{m}$. GIXRD patterns of quasi-2D perovskite thin films with different processing conditions in e) DMF RT, f) DMSO RT, g) Hot-cast technique, h) Doped $10 \% \mathrm{~K}^{+}$. i) Schematic diagram of parallel orientation for quasi-2D perovskite. $\mathrm{j}$ ) Schematic diagram of vertical orientation for quasi-2D perovskite. k) $\theta-2 \theta$ XRD 
patterns of quasi-2D perovskite thin films with different processing conditions. Inset shows their amplified XRD pattern at small diffraction angle. 1) FWHM and crystallite size of quasi-2D perovskite thin films with different processing conditions. 
(a)

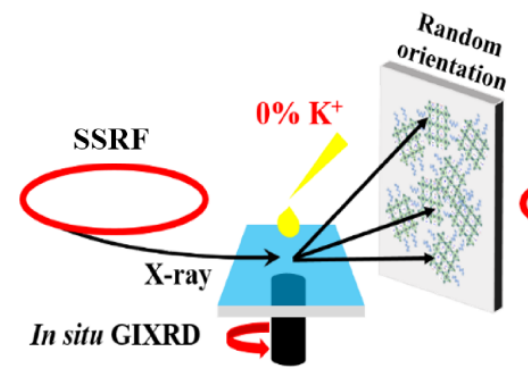

(c)

(d)

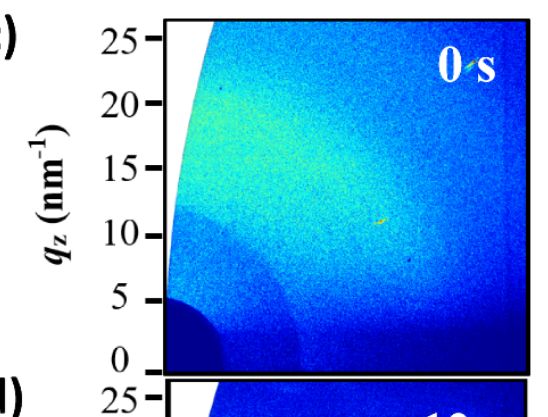

(e)

(f)
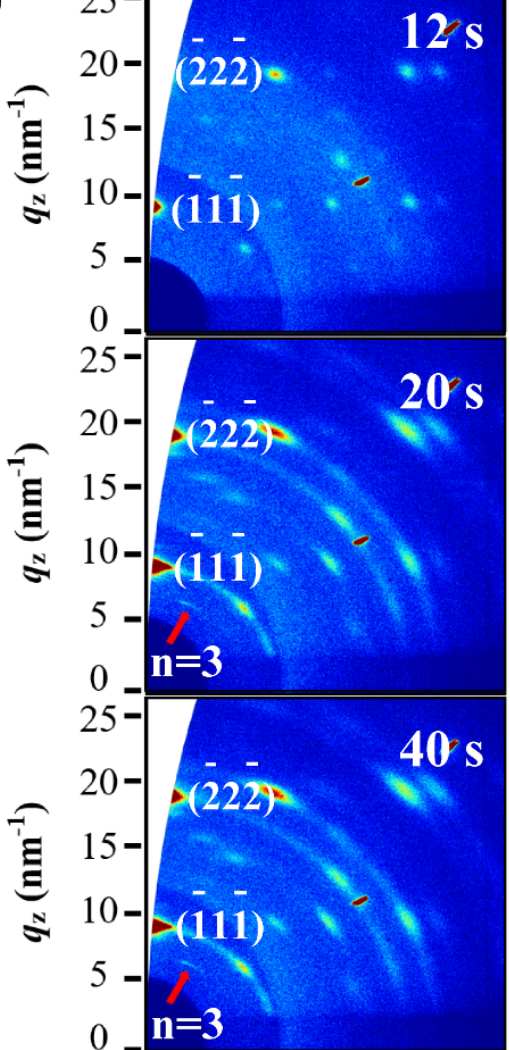

(g)

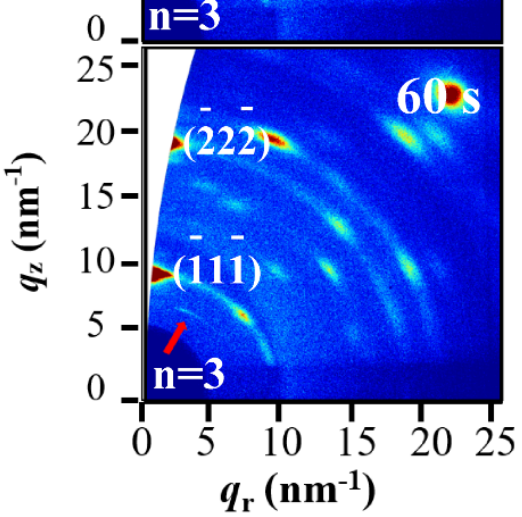

20
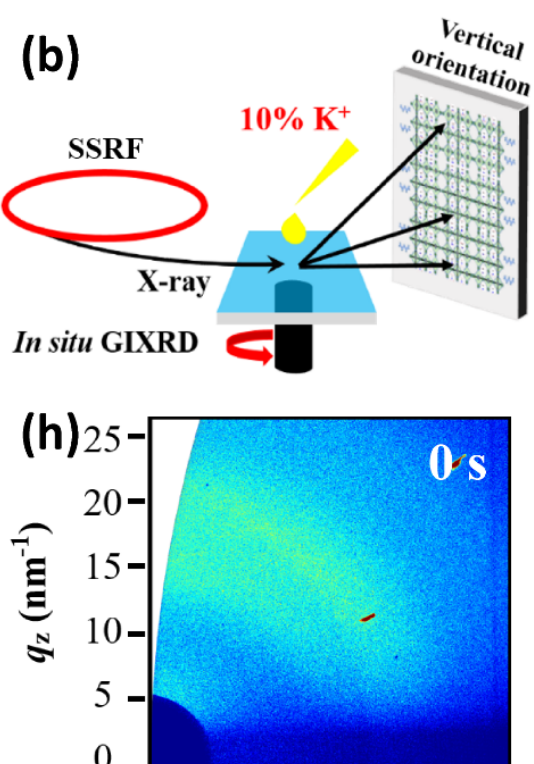

(i)

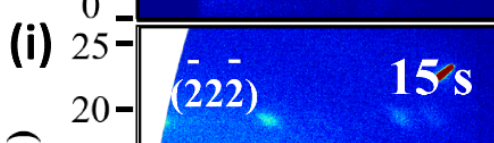

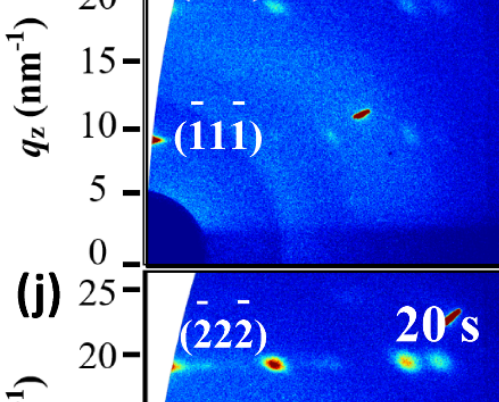

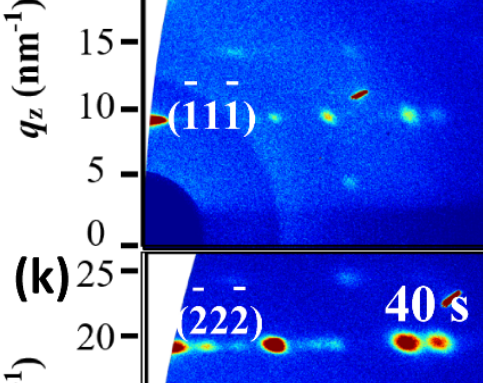

息 $15-\left(\begin{array}{ll}-\overline{1} \\ \approx\end{array}\right.$

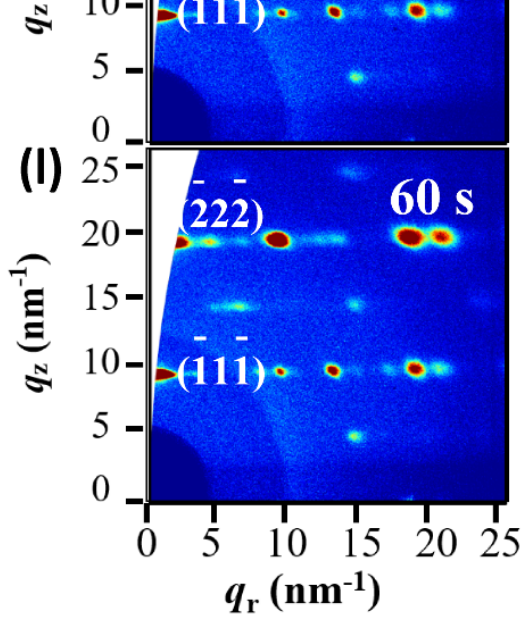

20 
Figure 2 a) Schematic diagram of in-situ synchrotron-based GIXRD characterization for doped $0 \% \mathrm{~K}^{+}$quasi-2D perovskite thin films. b) Schematic diagram of in-situ GIXRD characterization for doped $10 \% \mathrm{~K}^{+}$quasi-2D perovskite thin films. In-situ GIXRD patterns of doped $0 \% \mathrm{~K}^{+}$quasi-2D perovskite thin films in spinning coating process from $0 \mathrm{~s}$ to $60 \mathrm{~s}$. c) $0 \mathrm{~s}$, d) $12 \mathrm{~s}$, e) $20 \mathrm{~s}$, f) $40 \mathrm{~s}$, g) $60 \mathrm{~s}$. Red arrows represents low $n$-number intermediate phases. In-situ GIXRD patterns of doped $10 \% \mathrm{~K}^{+}$quasi-2D perovskite thin films in spinning coating process from $0 \mathrm{~s}$ to $60 \mathrm{~s}$. h) $0 \mathrm{~s}$, i) $15 \mathrm{~s}$, j) $20 \mathrm{~s}$, k) $40 \mathrm{~s}, 1) 60 \mathrm{~s}$. 
(a)

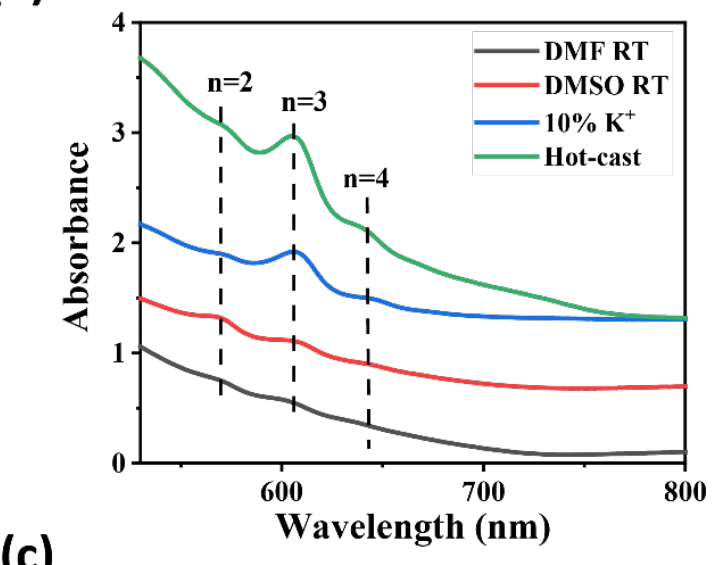

(c)

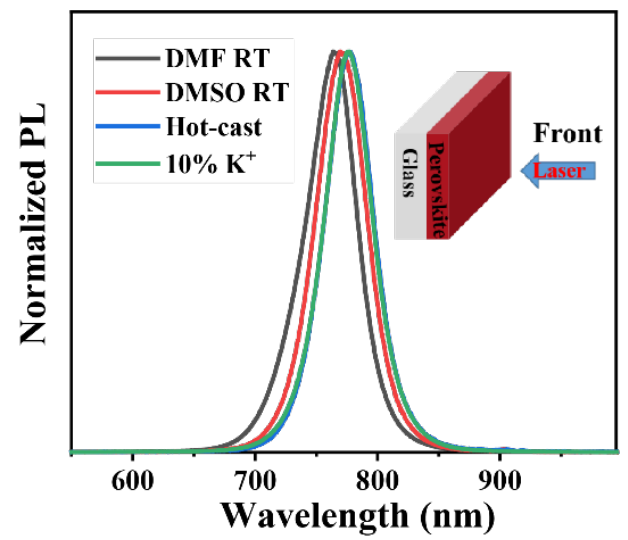

(b)
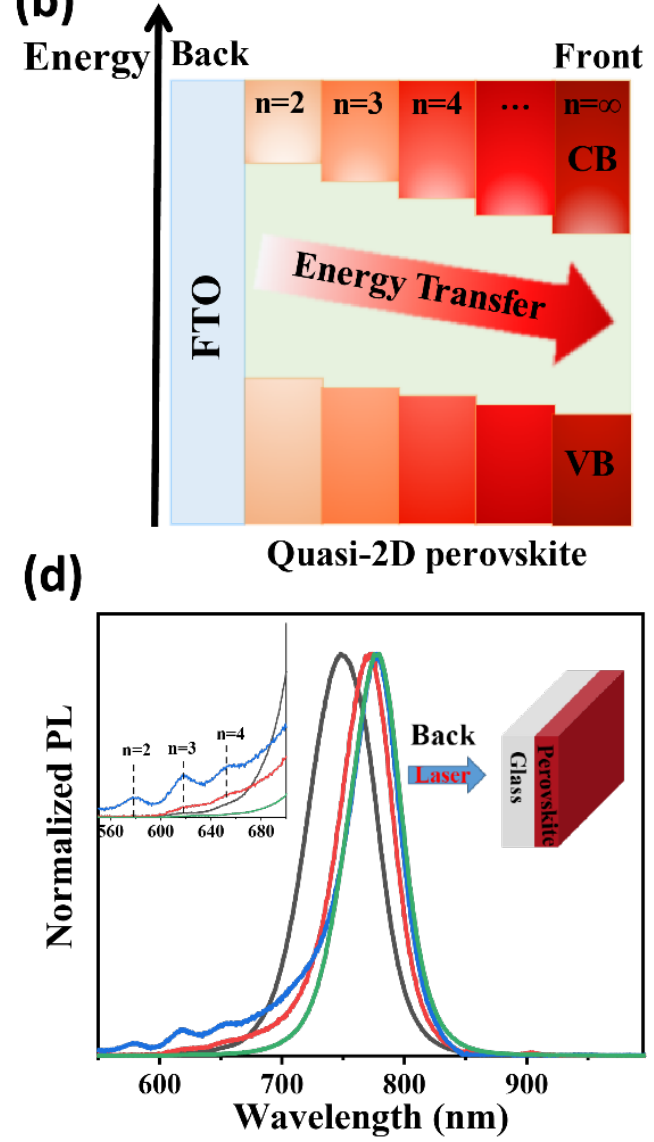

Figure 3 a) UV-vis absorption spectra for quasi-2D perovskite thin films with different processing conditions. b) Schematic diagram of energy transfer between different quasi-2D perovskite phase and 3D phase. c) Steady-state front side PL spectra of quasi2D perovskite thin films with different processing conditions. Inset shows the test direction from the front perovskite side. d) Steady-state rear side PL spectra of quasi2D perovskite thin films with different processing conditions. Right inset shows the test direction from the rear side. Left inset shows their amplified PL pattern at short wavelength. 
(a)

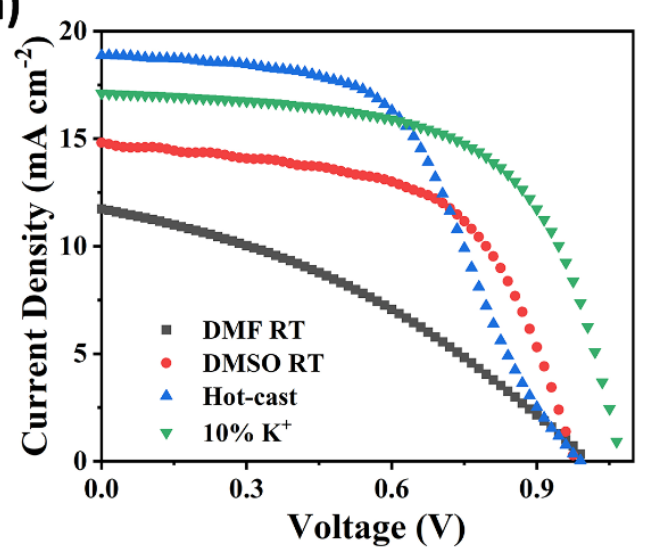

(b)

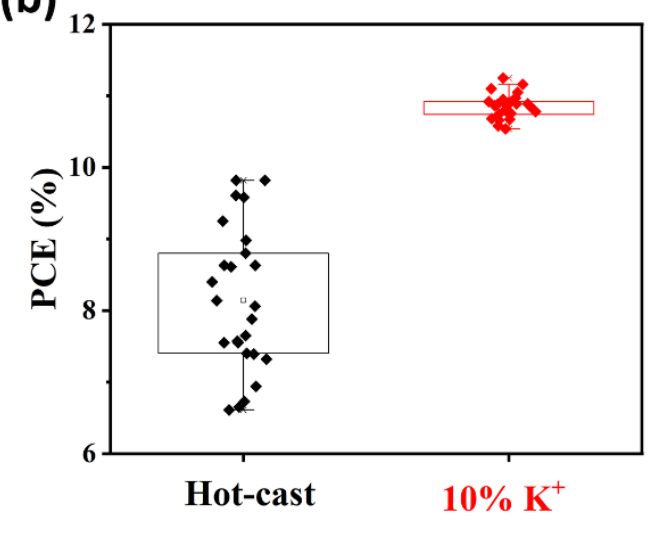

Figure 4 a) Typical $J-V$ curves of quasi-2D perovskite devices with different processing conditions under simulated AM $1.5 \mathrm{G}$ light illumination. b) $P C E$ distribution of solar cells with $10 \% \mathrm{~K}^{+}$and hot-cast technique. 
Table 1 Solar cell parameters for the quasi-2D perovskite devices with different processing conditions.

\begin{tabular}{ccccc}
\hline Sample & $V_{o c}(\mathrm{~V})$ & $J_{s c}\left(\mathrm{~mA} \mathrm{~cm}^{-2}\right)$ & $F F(\%)$ & $P C E(\%)$ \\
\hline DMF RT & 1.01 & 11.8 & 36.1 & 4.24 \\
DMSO RT & 0.99 & 14.8 & 57.2 & 8.46 \\
Hot-cast & 1.00 & 18.9 & 52.1 & 9.82 \\
$10 \% \mathrm{~K}^{+}$ & 1.08 & 17.1 & 62.3 & 11.3 \\
\hline
\end{tabular}




\section{The table of contents entry}

We discover that the incorporation of potassium ion into quasi-2D precursor solution can change the nucleation process via in-situ synchrotron-based grazing incident X-ray diffraction, which forms vertical oriented 2D phase without intermediate compound and reduces the distribution of low $n$-number phases.

\section{Revealing Crystallization Dynamics and Compositional Control Mechanism of}

\section{D Perovskite Film Growth by In-situ Synchrotron-based GIXRD}

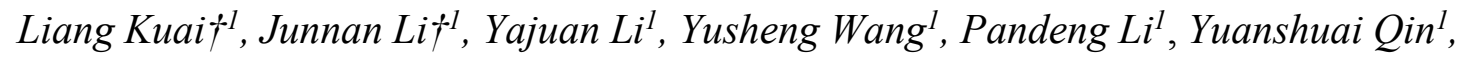

Tao Song ${ }^{1}$, Yingguo Yang ${ }^{2 *}$, Zhuoying Chen ${ }^{3}$, Xingyu $\mathrm{Gao}^{2}$, Baoquan Sun ${ }^{1 *}$

TOC

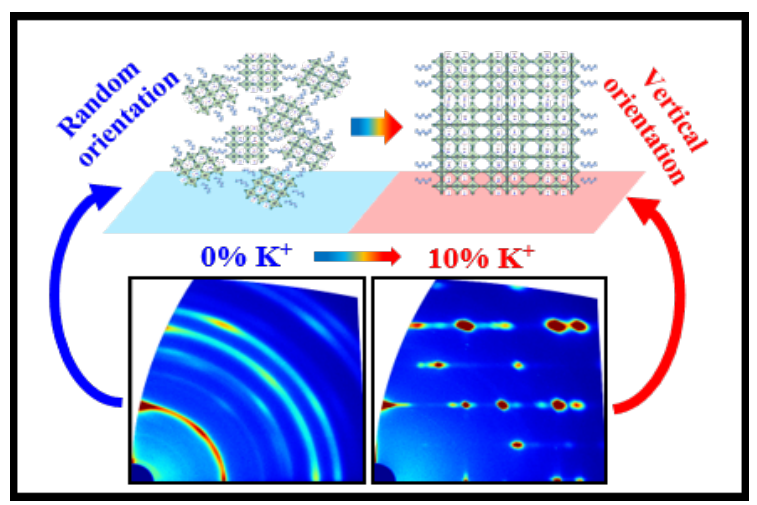

